\title{
The Watering Can Technique for Prevention of Postoperative Epidural Hematoma Recurrence
}

\author{
Matthew C. Tate ${ }^{1}$, Aaron Clark ${ }^{2}$, Michael W. McDermott ${ }^{3}$ \\ 1. Department of Neurosurgery, University of California, San Francisco 2. Department of Neurological \\ Surgery, University of California at San Francisco 3. Department of Neurological Surgery, University of \\ California, San Francisco
}

$\square$ Corresponding author: Matthew C. Tate, biomedmatt@yahoo.com

Disclosures can be found in Additional Information at the end of the article

\section{Abstract}

Background: Postoperative epidural hematomas (EDH) are relatively common occurrences following emergent or elective craniotomies. While a number of strategies have been reported for management of traumatic EDH, there is no consensus among surgeons regarding the most effective treatment of postoperative EDH.

Objective: We describe a novel surgical technique for treatment of postoperative EDH that has proved useful in the prevention of EDH recurrence.

Methods: A technique was developed using multiple linear arrays of dural tenting sutures to prevent EDH recurrence in the postoperative setting. Following removal of the EDH, bleeding points are identified and controlled. Dural tenting sutures are then placed in linear array over the entire exposed dural surface. Absorbable gelatin sponges are then placed between the dural tenting sutures in an alternating fashion. Holes corresponding to the dural sutures are drilled into the bone flap, which is fastened to the surrounding skull. Dural sutures are then secured to the overlying bone. The final appearance of the repair resembles a common gardening "watering can" (WC), hence, the proposed title of the technique.

Results: The WC technique for treatment of postoperative EDH is presented. Intraoperative photographs as well as an artist's rendition of the steps involved in the repair are detailed. Of the more than 20 cases of postoperative EDH repaired using the WC method, zero patients required return to the operating room for recurrence.

Conclusions: The WC technique for treatment of postoperative EDH provides a simple, effective strategy that may improve outcomes and limit morbidity relative to conventional methods.

Review began 11/07/2012

Published 11/08/2012

(c) Copyright 2012

Tate et al. This is an open access article distributed under the terms of the Creative Commons Attribution License CC-BY 3.0., which permits unrestricted use, distribution, and reproduction in any medium, provided the original author and source are credited.
Categories: Neurosurgery

Keywords: epidural hematoma, watering can, dural tenting sutures, hematoma, postoperative hematoma

\section{Introduction}

Postoperative EDH is relatively common following supratentorial craniotomy. In a large series specifically investigating postoperative EDH in 1,105 intracranial operations, the rate of EDH was $16 \%$, and the rate of clinically significant EDH requiring reoperation was $1 \%$ [1]. The vast 
majority of these EDH were found either beneath the bone flap or adjacent to the original surgical site. The rate of postoperative EDH appears to be related to the type of intracranial operation, with vascular and traumatic cases having the highest rates of symptomatic postoperative EDH [1-2]. In addition, larger craniotomies, such as those performed in trauma or epilepsy surgeries, are also associated with higher rates of postoperative EDH [3].

A number of techniques are used in the modern neurosurgical theater to prevent postoperative $\mathrm{EDH}$ at the initial operation. The most important technique is identification of bleeding points in the surgical field and meticulous hemostatic control with bipolar cautery and hemostatic agents. Another widely used approach is dural tenting sutures, which were first introduced by Dandy [4].

These tenting sutures, also commonly referred to as "tack-up" stitches, are generally placed along the periphery of the exposed dura with the goal of decreasing postoperative EDH by minimizing the potential epidural space via apposition of the dura to the bone. While recent studies suggest that tenting sutures may not be necessary for all intracranial operations (given improved hemostatic agents, instrumentation, and intraoperative blood pressure control) [2, 56], most surgeons still use dural tenting sutures for the majority of craniotomies. In addition, some surgeons use epidural drains to aid in prevention of postoperative EDH, though a recent study failed to show improvement in EDH rates with epidural drain placement after craniotomy for epilepsy [3].

While the issue of prophylactic use of dural tenting and epidural drainage remains controversial, particularly challenging surgical scenarios are treating postoperative EDH. In this setting, a single bleeding point may be difficult to identify, and it is common to see the entire dura "oozing". These EDH may represent surgical emergencies where the goal is to evacuate the EDH and prevent reaccumulation. Surgeons generally evacuate the EDH, address obvious bleeding sources with electrocautery, and then employ techniques similar to those performed at initial craniotomy (tenting sutures, hemostatic agents, closed suction drains). Unfortunately, there are few reports on specific techniques to address postoperative EDH. Park, et al. demonstrated improved outcomes for patients with a moderate-sized postoperative EDH beneath the bone flap after thrombolytic infusion via a closed suction epidural drain placed during the initial craniotomy [7]. There are no recent reports, to our knowledge, describing specific techniques for operative treatment of large, symptomatic postoperative EDH. We report a surgical technique for the removal and prevention of recurrent postoperative epidural hematoma that involves multiple rows of dural tenting sutures with interspaced absorbable hemostatic agents. The corresponding holes in the bone flap for the sutures resemble the spout of a "watering can" (WC) and we have coined this term for the technique.

\section{Technical Report}

First, a craniotomy is performed and the EDH is evacuated in a standard fashion. Bleeding points are identified and controlled with bipolar electrocautery and routine hemostatic agents. Once bleeding is controlled, vertical columns of dural tack-up sutures are placed every $1 \mathrm{~cm}$ covering the entire exposed dural surface ( -4 finger's breadth or $1 \mathrm{~cm}$ between each row). Within a given row, the distance between sutures is approximately $5 \mathrm{~mm}$ (Figures $1 \mathrm{~A}, 2 \mathrm{~A}$ ). Next, $1 \mathrm{~cm}$ wide strips of gelatin sponge (Gelfoam ${ }^{\circledR}$, Pfizer, New York, NY) are trimmed to the appropriate length and placed between the rows of tenting sutures, such that the entire exposed epidural surface is addressed (Figures $1 B, 2 B$ ). Vertical drill hole pairs corresponding to tenting suture rows are placed in the overlying bone flap, which at this point resembles a common watering can used in gardening (Figures 1C, 1D) (Figure 2C) (Figure 3). A 7-French suction is then employed to facilitate guidance of both ends of each tenting suture through its corresponding drill holes in the bone flap (Figures 1E, 2D). The bone flap is then secured to the adjacent bone with titanium screws and the dural tack-up sutures are cinched to the bone flap, 


\section{Cureus}

tied, and cut. A 1/4-inch Hemovac drain is placed in the subgaleal space under suction and left in place for 24 hours. The galea is closed with a running 2-0 absorbable suture, followed by a running 4-0 nylon suture for the skin layer.

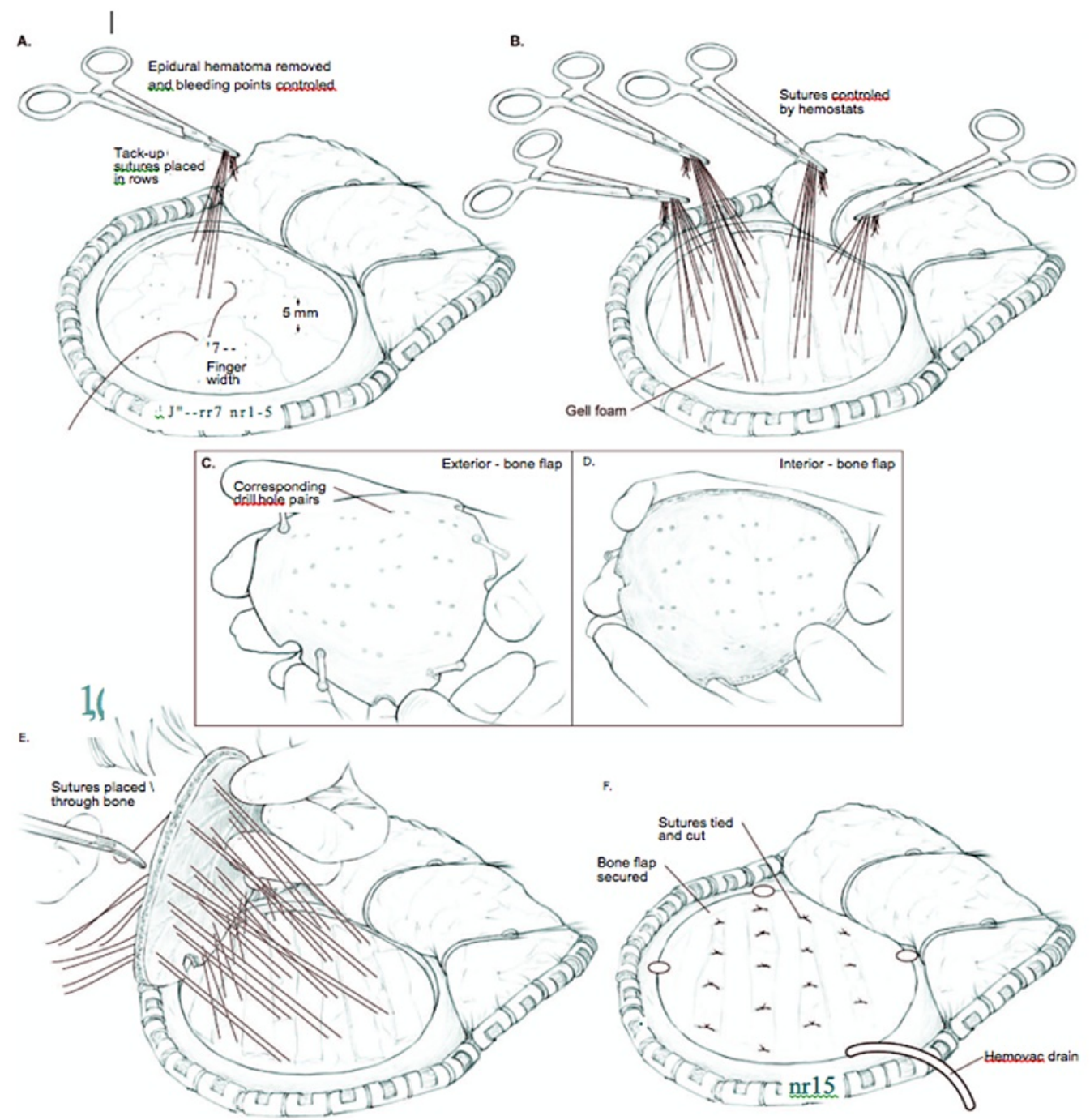

\section{FIGURE 1: Watering can repair.}

Following hematoma removal, linear arrays of interrupted 4-0 nylon dural sutures are placed and secured with a hemostat (A). Strips of gelfoam are placed in alternating columns between rows of dural tack-up sutures (B). Holes are drilled in the bone flap corresponding to dural sutures $(C, D)$. Dural suture ends are then guided through corresponding holes in bone flap and cinched (E). The bone flap is then secured with standard fixation hardware followed by placement of a subgaleal drain (F). 


\section{Cureus}

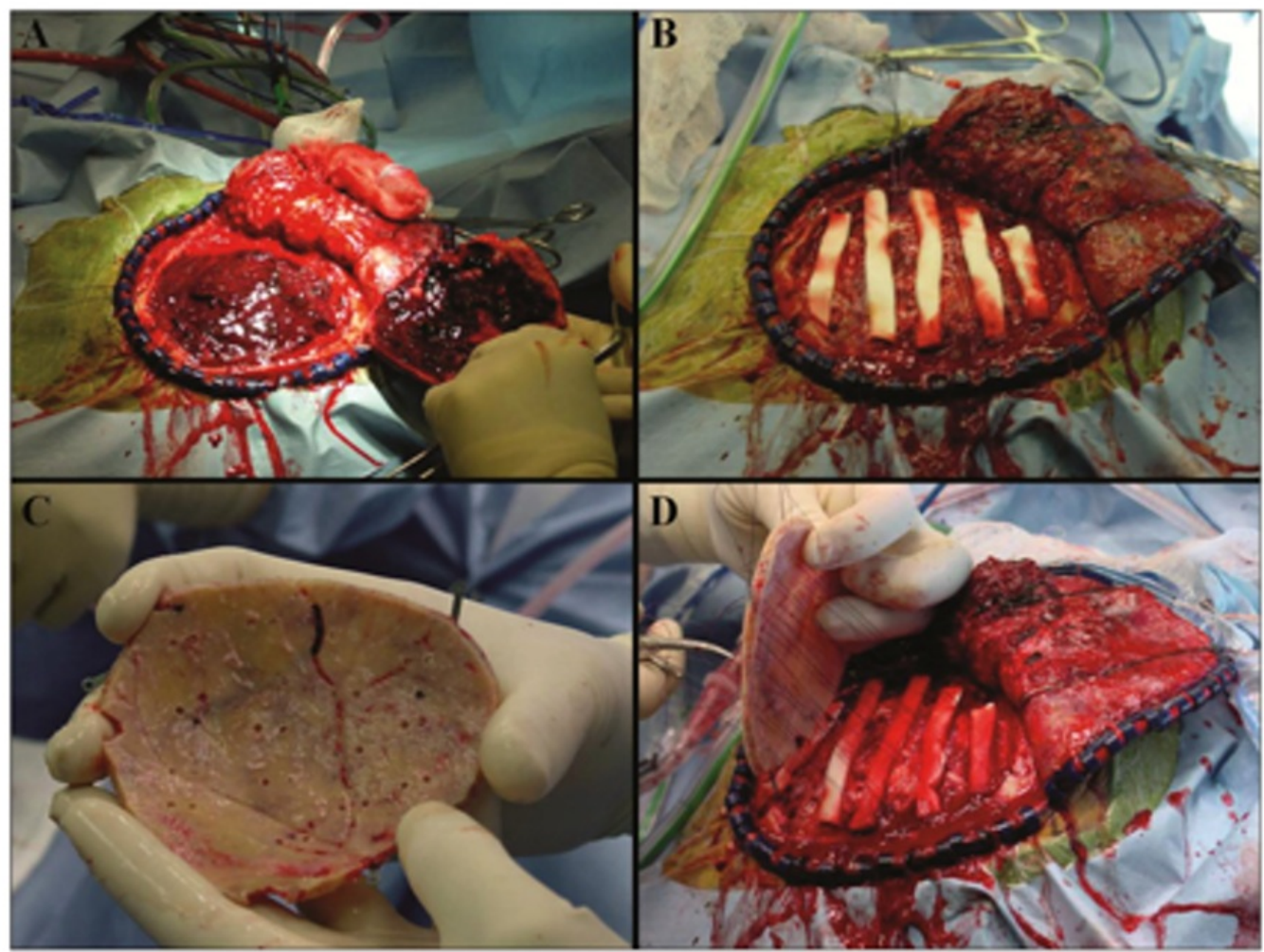

\section{FIGURE 2: Intraoperative photos of stages of watering can repair.}

First, a re-do craniotomy is performed to expose the epidural hematoma (A). 4-0 braided nylon dural tack-ups and gelatin sponge strips are placed in alternating columns $1 \mathrm{~cm}$ apart (B). Holes for accommodation of dural stitches are drilled in the bone flap (C). Dural sutures are fed through the bone flap prior to fixation (D).

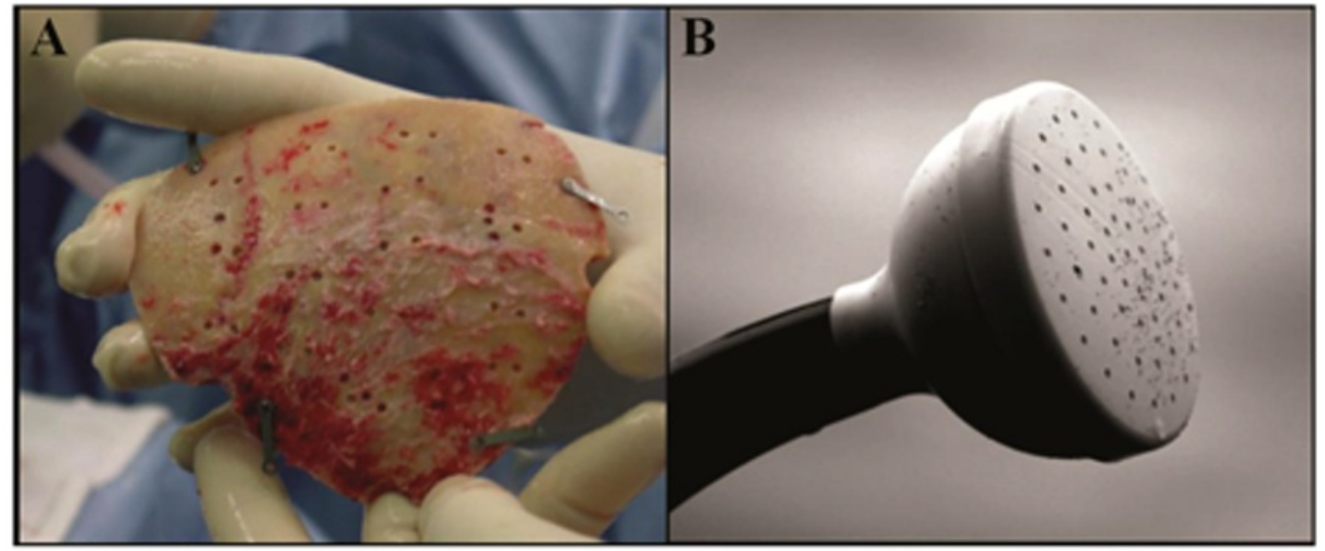

FIGURE 3: Origin of "watering can" nomenclature.

Intraoperative external view of bone flap (A) which is similar in appearance to a standard watering can used for gardening $(B)[8]$.

\section{Results}


The WC technique for treatment of post-craniotomy EDH has proved useful in our institution and has been used in over 20 patients since 1990. The increase in operative time for the WC repair relative to standard dural tenting sutures is approximately 20 minutes. No intraoperative complications were noted in the series receiving this repair. Importantly, no recurrent EDH necessitating return to the operating room was observed in those patients receiving the WC repair.

\section{Discussion}

In this report, we discuss a technique used by the senior author for the treatment of postoperative EDH. The WC method has a number of advantages over traditional surgical treatments for EDH and postoperative EDH. The additional number of tenting sutures, as compared to standard closure techniques with interrupted sutures at the periphery and one or two central sutures, serves to dramatically decrease the potential epidural space. The increased dural surface area in contact with gelatin sponge also acts to decrease the potential space for EDH accumulation as well as providing a substrate, which hastens clot formation [9] and thus continues to promote hemostasis following closure. The collagen sponge and increased tenting suture density further serve to increase the local resistive pressure, which may serve to counteract venous bleeding from the bone and dura. The WC repair may also be superior to epidural drain strategies due to reduction of potential space and promotion of hemostasis rather than simply diverting accumulating blood. Though a recent study demonstrated efficacy of thrombolytic infusion via epidural drains for postoperative EDH, this strategy is limited to smaller EDH and requires epidural drain placement at initial surgery, which is not routine. Thus, the WC repair is a more robust and definitive management strategy for the symptomatic patient with a postoperative EDH. From a cost-benefit perspective, the materials needed for the WC repair (nylon sutures, Hemovac drain, collagen sponge, drill, standard craniotomy fixation set) are already available on the standard operative setup and are relatively inexpensive. Operative time is only moderately longer but may be offset by the lack of need for repeated follow-up imaging when there is reaccumulation and certainly, when there is need for an additional surgical procedure. Finally, there is a psychological benefit to the surgeon in minimizing EDH recurrence in these difficult and often emergent operative scenarios where the WC closure would be employed.

As with any new surgical approach, there are potential disadvantages. Given that with any dural stitch there is a chance of injury/bleeding from damage to underlying cortical vessels [10], the absolute chance would increase with the WC repair given that more stitches are employed. However, refinements in techniques for placement of dural stitches have decreased this risk [11], and the majority of these injuries is evident and remedied upon immediate inspection or prior to bone flap replacement. Another potential disadvantage is the increase in operative time. While this may be a concern during the initial cases needed to learn the procedure, once the technique has been mastered, we estimate that the additional operative time is approximately 10-20 minutes. Also, the addition of collagen sponge has the possibility of exerting undue mass effect locally but this is limited by the numerous tack-up stitches. We argue that use of a standard thin collagen sponge exerts only minimal mass effect which in fact is likely beneficial by increasing the local pressure gradient that must be overcome for venous bleeding to ensue. We have had no significant complications related to collagen sponge usage in our series.

The WC method described in this study was developed for treatment and prevention of recurrence in the setting of postoperative EDH. However, based on first principles and given the minimal morbidity profile of the WC method, it is certainly reasonable to consider its use in initial craniotomies, particularly for cases in which the occurrence of EDH is a major concern (vascular, trauma, coagulopathy, and large craniotomies with increased epidural potential space). Optimization of the WC method is also being considered. Questions that remain include 
the optimal suture:gelfoam ratio and the utility of incorporation of other hemostatic agents.

\section{Conclusions}

The WC technique for repair and prevention of recurrent EDH has proven useful in a single surgeon's experience over the past decade. This technique provides a simple, effective strategy for surgical management of postoperative EDH that may improve outcomes and limit morbidity versus conventional methods. In addition, the method may be reasonable for initial craniotomy in cases with a high likelihood of postoperative EDH.

\section{Additional Information \\ Disclosures}

Human subjects: All authors have confirmed that this study did not involve human participants or tissue. Animal subjects: All authors have confirmed that this study did not involve animal subjects or tissue. Conflicts of interest: In compliance with the ICMJE uniform disclosure form, all authors declare the following: Payment/services info: All authors have declared that no financial support was received from any organization for the submitted work. Financial relationships: All authors have declared that they have no financial relationships at present or within the previous three years with any organizations that might have an interest in the submitted work. Other relationships: All authors have declared that there are no other relationships or activities that could appear to have influenced the submitted work.

\section{Acknowledgements}

The authors wish to acknowledge Kenneth Probst (Xavier Studio, San Francisco, CA) for assistance with artwork.

\section{References}

1. Fukamachi A, Koizumi H, Nagaseki Y, Nukui H: Postoperative extradural hematomas: Computed tomographic survey of 1105 intracranial operations. Neurosurg. 1986, 19:589-593. 10.1097/00006123-198610000-00013

2. Swayne OB, Horner BM, Dorward NL: The hitch stitch: An obsolete neurosurgical technique?. Br J Neurosurg. 2002, 16:541-544. 10.1080/02688690209168357

3. Guangming Z, Huancong Z, Wenjing Z, Guoqiang C, Xiaosong W: Should epidural drain be recommended after supratentorial craniotomy for epileptic patients?. Surg Neurol. 2009, 72:138-141. 10.1016/j.surneu.2008.06.014

4. Lewis D: Practice of Surgery. WF Prior, Hagestown, MD; 1930.

5. Winston KR: Dural tenting sutures in pediatric neurosurgery. Pediatr Neurosurg. 1998, 28:230-235. 10.1159/000028656

6. Winston KR: Efficacy of dural tenting sutures. J Neurosurg. 1999, 91:180-184. 10.3171/jns.1999.91.2.0180

7. Park J, Kim GJ, Hwang SK: Thrombolytic evacuation of post-craniotomy epidural haematomas using closed suction drains: A pilot study. Acta Neurochir. 2008, 150:359-366. 10.1007/s00701-007-1487-4

8. Wikimedia. (2006). Accessed: December 30, 2006: http://commons.wikimedia.org/wiki/File:Watering_Can_Spout.jpg.

9. Guralnick WC: Absorbable gelatin sponge and thrombin in oral surgery. Am J Orthod. 1946, 32:792-794. 10.1016/0096-6347(46)90041-5

10. Aoki N: Acute subdural hematoma during tack-up suture of the dura mater--Case report . Neurol Med Chir. 1988, 28:994-995. 10.2176/nmc.28.994

11. Apuzzo MJ: Brain surgery: Complication avoidance and management. Churchill Livingstone, New York; 1993. 10.1001/jama.1993.03510160110039 